Ann. Génét. Sél. anim., I977, 9 (3), 393-405.

\title{
Optimisation du progrès génétique sur la prolificité chez le Lapin
}

\author{
G. MATHERON et R. ROUVIER \\ Station d'Améliovation génétique des Animaux \\ Centre de Recherches de Toulouse, I.N.R.A., \\ B.P. I2. 3I320 Castanet-Tolosan
}

\begin{abstract}
Résumé
Une méthode de sélection originale du lapin pour accroître la productivité numérique des femelles, dans le cas d'un troupeau fermé d'effectif limité et de générations séparées, est présentée et discutée. La méthode d'indexation des lapines basée sur la connaissance des 3 premières portées de chaque femelle, sur le renouvellement du noyau de sélection à partir des issues des deux premières portées, conduit à la valeur la plus grande de l'espérance $(R)$ du progrès génétique annuel sur le nombre de nés vivants par portée (ou de sevrés). On trouve en effet $\mathrm{R}=0,43$, pour la voie mère-fille de transmission du progrès, ce qui est de l'ordre du double de ce qui avait été trouvé pour un troupeau avec des générations chevauchantes, lorsque l'on ne prenait pas en compte l'information des pleines sœurs et de la mère de la femelle candidate à la sélection. L'on a discuté les points suivants : sélection en souche pure pour l'amélioration des lapines croisées; choix des souches à sélectionner; plan d'accouplement et méthode utilisés. Ceux-ci doivent être à la fois optimum dans le court terme et permettre les études à moyen terme : mesure de l'évolution génétique des populations; estimation des paramètres génétiques; efficacité d'une sélection récurrente.
\end{abstract}

Chez le Lapin, comme chez les autres espèces de mammifères domestiques producteurs de viande, la productivité numérique annuelle des femelles est un facteur important de la rentabilité de l'élevage mesurée au niveau du couple mèreproduits (MoAv, I966). Après avoir étudié, en race pure et en croisement, les performances d'animaux de 6 races élevées au laboratoire, nous avons défini la méthode de sélection en race pure de deux d'entre elles. Il s'agit d'une sélection de deux souches (troupeaux fermés d'effectif limité), l'une donnant des mâles, l'autre des femelles qui accouplées en croisement doivent produire des lapines " hybrides " à forte productivité numérique.

Cela nous a conduit, après avoir choisi les animaux des deux lignées et le meilleur sens du croisement, à chercher le plan d'accouplement et la méthode de sélection permettant d'optimiser le progrès génétique attendu dans chacune d'elles, pour le nombre de lapereaux nés vivants ou sevrés, par lapine et par an. 
Une telle expérimentation doit tenir compte des caractéristiques biologiques et zootechniques du lapin, ainsi que des objectifs d'amélioration génétique de cette espèce. Il nous a paru utile, dans le présent article, d'indiquer et discuter le principe de la méthode de sélection utilisée, compte tenu de son originalité dans le cas du lapin. Nous discuterons aussi la détermination et l'utilisation de la quantité d'information optimale pour améliorer la valeur génétique d'une des composantes de la productivité numérique (taille de portée), d'un troupeau fermé d'effectif limité conduit en souche pure avec un nombre de reproducteurs décroissant au cours du temps dans chaque génération.

\section{I. - Matériel animal}

Les deux types génétiques sélectionnés à l'I.N.R.A. et servant de base à la production de femelles croisées ou "hybrides " sont de deux origines différentes. La première (souche A I066) fut constituée en I970 à partir d'animaux Californiens Anglais et Grands Russes; ces derniers provenant d'élevages fermiers du Maine et Loire. Six générations de conduite en troupeau fermé ont permis de fixer les gènes intéressants, d'homogénéiser la population et d'en augmenter l'effectif. Ce troupeau fermé diffuse depuis r976 les reproducteurs du sexe mâle utilisés par les éleveurs multiplicateurs. Les femelles proviennent de l'autre souche (A I077), dont nous exposons ici le protocole détaillé de sélection.

Quelques animaux Néo-Zélandais blancs importés de Californie par Rouvier en I968, permirent de disposer en 1973 d'une population de lapines homogène, d'effectif suffisant pour y démarrer une sélection. Ces deux populations de roo et I 6 reproductrices respectivement, sont conduites en troupeau fermé, en simultanéité, avec un rythme de reproduction semi-intensif (présentation des lapines au mâle ro jours après la mise bas) et ont des intervalles de générations compris entre 9 et $\mathrm{I} 2$ mois.

\section{II. - La méthode de sélection}

Poujardieu et Rouvier (I972) ont montré l'intérêt, pour des caractères maternels de faible héritabilité (tels que la taille de portée des mammifères, ou la production d'œufs des poules) d'une sélection combinée basée sur les performances individuelles et des collatéraux. Une telle méthode de sélection, discutée déjà par Lush (I947), OsBorne (I957) chez la Poule, permet d'accroître la précision de l'estimation de la valeur génétique additive d'une reproductrice sans accroître l'intervalle de génération. La méthode de sélection utilisée pour augmenter la productivité numérique et plus particulièrement la taille de portée des lapines, repose sur ces considérations. Dans le cas du lapin, il faut tenir compte d'une "fonte " du cheptel de reproductrices qui peut être de l'ordre de 4 à 5 p. Ioo par mois. L'objectif premier étant d'améliorer la taille de portée, il fallait rechercher, en tenant compte de la "fonte " de cheptel, l'optimum entre la quantité d'information sur chaque femelle (nombre de portées, nombre de collatérales) et la longueur de l'intervalle de génération, le pourcentage de lapines sélectionnées, dans l'hypothèse d'une conduite suivant des générations séparées. Cette hypothèse a été prise essentiellement pour des raisons expérimentales : comparaison, sur leur valeur génétique estimée, de lapines contemporaines. 


\section{I. - Plan d'accouplement et mode de conduite du troupeau}

Le troupeau est conduit en bandes de reproduction séparées, chaque bande constitue une génération. A chaque génération un effectif constant de 196 femelles est mis en place avec un lot de 42 mâles. La méthode de sélection prévoit de sélectionner un certain pourcentage de ces femelles d'après leurs résultats de taille de portée sur un certain nombre de mises bas. Les femelles ainsi choisies donnent alors les fils et les filles de renouvellement. Le nombre d'ancêtres fondateurs de la souche A I077 fait qu'à chaque génération les I 96 femelles mises en comparaison sont réparties en $\mathrm{I}_{4}$ familles de $\mathrm{I} 4$ pleines sœurs et demi-sœurs de père. Cette répartition permet de faire sur ce troupeau I4 groupes de reproduction chacun

\section{TABLEAU I}

Réalisation des groupes de reproduction à partir des origines familiales Realisation of reproductive groups from family origins

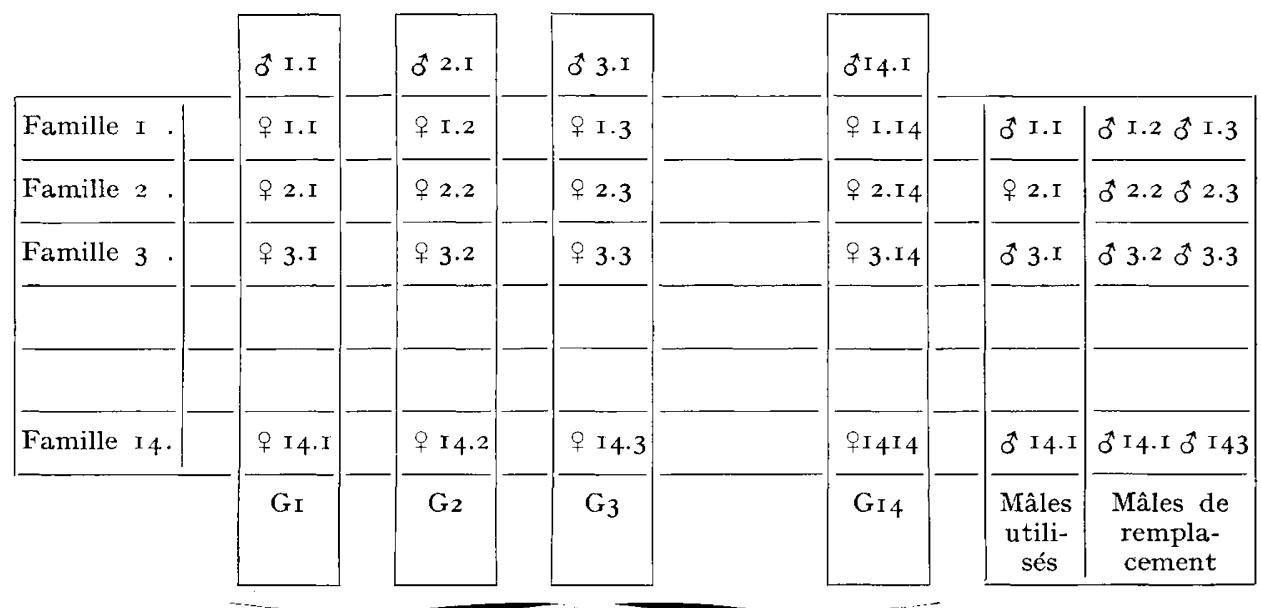

Groupes de reproduction

constitué de I mâle (plus 2 suppléants) et de I4 femelles. Celles-ci sont tirées au hasard, à raison d'une par famille, parmi le lot de Ig6 femelles (tabl. no I). Ce plan d'accouplement, ressemblant à celui discuté par Lerner (I958) chez la Poule, en diffère toutefois : les groupes de reproducteurs sont ici constitués avant la connaissance des valeurs génétiques des différents reproducteurs d'une génération. Il en résulte que les filles de renouvellement, ainsi que les fils, sont strictement sélectionnés d'après l'indice de leur mère, avec une pression de sélection différente suivant ces deux sexes puisqu'il faut moins de mâles que de femelles. Si le progrès génétique lié au choix des femelles de renouvellement peut s'exprimer d'une génération à la suivante, celui lié aux mâles de renouvellement ne s'exprime que chez leurs filles. Nous nous limiterons dans un premier temps à discuter le progrès génétique par génération lié au choix des femelles de renouvellement, donc à la voie mère-fille. 


\section{2 - L'indexation des reproductrices}

Dans le groupe d'animaux ainsi mis en reproduction il faut à chaque génération isoler les meilleurs génotypes pour le caractère dont on veut augmenter le niveau génétique moyen. Pour cela il faut connaître les aptitudes des femelles mises en place en début de bande. Les performances prises en compte pour classer les femelles sont celles concernant les effectifs de lapereaux nés et sevrés, en moyenne par portée, par les femelles ayant fait un nombre $k$ de portées dans une période de temps défini, depuis la mise en reproduction. Les femelles qui n'ont pas fait ces $k$ portées au moins sont écartées de l'indexation.

La faible héritabilité $\left(h^{2}=0,05\right.$ à $\left.0, I_{5}\right)$ des caractéristiques d'une portée conduit à prendre en compte, pour prédire la valeur génétique additive d'une femelle avec le plus de précision possible, le maximum d'information dont on dispose sur cet animal. C'est pourquoi l'indice utilisé combine la performance, de la femelle ( $k$ portées), celle de ses pleines sœurs ( $s_{k}$ femelles ayant chacune $k$ portées connues) et celle de sa mère ( $k^{\prime \prime}$ portées).

Si l'on appelle Fijl la performance de la $l$ e portée de la $j$ e femelle de la $i$ e famille de pleines sœurs, Aij la valeur génétique additive de cette femelle et Mil la performance de la $l e$ portée de la mère du $i$ groupe de pleines sœurs, l'indice s'écrit pour une femelle (Lush, I947; Robertson, I955; Poujardieu et Rouvier, I972) :

$$
\mathrm{A} i j=\beta_{1}(\mathrm{~F} i j .-\mathrm{F} i . .)+\beta_{2}(\mathrm{~F} i \ldots-\mathrm{F} \ldots)+\beta_{3}(\mathrm{M} i .-\mathrm{M} \ldots)
$$

Si l'on appelle $s_{k}$, le nombre de pleines sœurs de la famille dont fait partie la femelle $i j$, et $\Gamma$ la matrice de variances-covariances des prédicteurs, les valeurs de $\beta_{1}, \beta_{2}, \beta_{3}$ sont données par :

$$
\left(\begin{array}{l}
\beta_{1} \\
\beta_{2} \\
\beta_{3}
\end{array}\right)=\Gamma^{-1} \times\left(\begin{array}{cc}
\left(s_{k}-\mathrm{I}\right) / 2 & s_{k} \\
\left(s_{k}+\mathrm{I}\right) / 2 & s_{k} \\
\mathrm{I} / 2 &
\end{array}\right) \times \sigma_{\mathrm{A}}^{2}, \quad \sigma_{\mathrm{A}}=\begin{aligned}
& \text { variance génétique } \\
& \text { additive du caractère. }
\end{aligned}
$$

La précision de l'indice de cette femelle s'écrit alors :

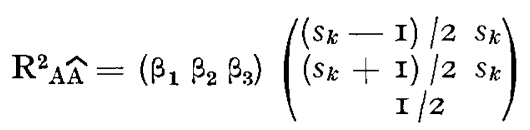

\section{III. - Optimisation du progrès génétique annuel}

Comme le rapelle Orlivier (I974), lorsque la sélection est la même dans les deux sexes, le progrès génétique attendu par unité de temps, $R$, est fonction de 4 paramètres : l'intensité de sélection $(i)$, l'intervalle de génération $(t)$, la corrélation $R_{A} \widehat{A}$ entre la valeur génétique additive $A$ que l'on veut améliorer et celle estimée par l'indice de sélection A, l'écart type génétique $\sigma_{\mathrm{A}}$.

$$
\mathrm{R}=\frac{i}{t} \times \mathrm{R}_{\mathrm{AA}} \times \sigma_{\mathrm{A}}
$$


Cet auteur a recherché le maximum de $i / t$ et de $i \mathrm{R}_{\mathrm{AA}} / t$ en fonction de la durée de vie reproductive des reproducteurs. Il a envisagé différents protocoles de sélection (sélection massale, sélection sur descendance, sélection de caractères maternels avec performances répétées) applicables à des troupeaux produisant en continu. Pour une population à générations séparées, FALCONER (I960) avait illustré la variation de $i / t$ en fonction de $i$ et de $t$ par un exemple adapté à la souris.

L'étude présente se distingue des précédentes par le mode de conduite du troupeau, et par la méthode d'indexation.

Comme dans l'exemple de FALCONER, les générations sont séparées, mais la fonte du cheptel a pour conséquence de diminuer l'intensité de sélection si l'on augmente le nombre $k$ de portées prises en compte dans l'indice. Par ailleurs nous avons envisagé un choix des reproductrices qui tient compte des performances des collatérales, ce qui permet d'accroître $R_{A} \widehat{A}$ sans augmenter l'intervalle de génération. Enfin, nous avons seulement considéré la transmission du progrès génétique par la voie mère-fille, et sur une seule génération. En effet, les femelles sélectionnées comme mères à mâles dans la génération o conduisent à des mâles améliorateurs utilisés en génération I dont l'apport au niveau génétique moyen du troupeau ne se fera sentir que sur les femelles de la génération 2. Ce n'est qu'expérimentalement que nous étudierons ce processus dynamique des effets de la sélection. Les résultats sur le progrès présenté ici sont à prendre comme des valeurs par défaut puisque la voie mère-fils, bien qu'avec un certain retard, contribue à l'amélioration du caractère.

\section{I. - Définition des paramètres}

Chez la lapine, la mise en reproduction peut se faire à l'âge de 5 mois; l'intervalle moyen entre deux mises bas successives a été pris égal à 2 mois. Nous considérons qu'il y a en moyenne, par portée, en tant qu'animaux utiles comme futurs reproducteurs, soit 2 mâles et 2 femelles, soit 3 mâles et 3 femelles, en fonction de la prolificité et de la viabilité du troupeau.

On peut à la fois tenir compte de l'information obtenue sur $k$ portées successives de chaque femelle, à partir de la première, et renouveler le cheptel à partir d'animaux issus de $k^{\prime}$ portées. En prenant pour $k$ et $k^{\prime}$ des valeurs comprises entre I et 4 , on obtient le tableau 2 qui fournit la description des ro cas que nous avons considérés avec les intervalles de génération auxquels ils conduisent.

La valeur de l'intensité de sélection $(i)$ est déterminée par le taux de sélection (P). Celui-ci est le rapport du nombre de femelles sélectionnées au nombre de femelles indexées. Nous faisons l'hypothèse que l'effectif du troupeau de sélection est constant à la mise en reproduction, soit $\mathrm{N}$. Nous tenons partiellement compte de la fonte du cheptel (élimination de femelles, ou mortalité). Nous nous basons en cela sur les observations zootechniques faites dans les élevages rationnels de production. Sur le troupeau de $\mathrm{N}$ femelles mises en places il y en a ${ }^{\prime}{ }_{k}$ qui font $k$ premières portées et qui sont donc indexables si l'indice est calculé sur le lot de femelles ayant fait au moins $k$ portées. Nous avons adopté une formulation qui rend compte d'une fonte de cheptel de $\mathrm{I}_{5} \mathrm{p}$. Ioo avant la première mise bas et de Io p. Ioo pour les portées suivantes, soit :

$$
\frac{\mathrm{N}^{\prime}{ }_{k}}{\mathrm{~N}}=\mathrm{Q}^{\prime}{ }_{k}=(\mathrm{I}-\mathrm{O} . \mathrm{I} 5)(\mathrm{I} \rightarrow \mathrm{O} . \mathrm{IO})^{k-1}
$$


TABLEAU 2

Les différents cas comparés et les intervalles de génération $(t)$ auxquels ils conduisent Different compared cases and generation intervals they induced

\begin{tabular}{|c|c|c|c|c|}
\hline $\begin{array}{l}\text { Nombre de Portées } \\
\text { de Renouvellement }\left(k^{\prime}\right)\end{array}$ & I & 2 & 3 & 4 \\
\hline $\begin{array}{c}\text { Nombre de Portées } \\
\text { d'information }(k) \\
\text { I }\end{array}$ & $\begin{array}{l}\text { Cas } \mathrm{n}^{0} \mathrm{I} \\
t=6 \text { mois }\end{array}$ & & & \\
\hline 2 & $\begin{array}{l}\text { Cas } \mathrm{n}^{\circ} 2 \\
t=6 \text { mois }\end{array}$ & $\begin{array}{l}\text { Cas } \mathrm{n}^{\circ} 3 \\
t=8 \text { mois }\end{array}$ & & \\
\hline 3 & $\begin{array}{l}\text { Cas } \mathrm{n}^{\circ} 4 \\
t=6 \text { mois }\end{array}$ & $\begin{array}{l}\text { Cas } \mathrm{n}^{0} 5 \\
t=8 \text { mois }\end{array}$ & $\begin{array}{c}\text { Cas } \mathrm{n}^{\circ} 6 \\
t=10 \text { mois }\end{array}$ & \\
\hline 4 & $\begin{array}{l}\text { Cas } \mathrm{n}^{\circ} 7 \\
t=8 \text { mois }\end{array}$ & $\begin{array}{l}\text { Cas } \mathrm{n}^{\circ} 8 \\
t=10 \text { mois }\end{array}$ & $\begin{array}{l}\text { Cas } \mathrm{n}^{0} 9 \\
t=\mathrm{I} 2 \text { mois }\end{array}$ & $\begin{array}{l}\text { Cas } n^{0} \text { 1o } \\
t=\mathrm{I} 4 \text { mois }\end{array}$ \\
\hline
\end{tabular}

Parmi les $\mathrm{N}$ femelles mises en testage, $\mathrm{N}^{\prime \prime}{ }_{k}$ sont sélectionnées. Le taux de sélection $\mathrm{P}_{k}$ est alors de $\mathrm{P}_{k}=\frac{\mathrm{N}^{\prime \prime} k}{\mathrm{~N}^{\prime}{ }_{k}}$

Le nombre moyen de pleines sœurs $s_{k}$ dans une famille est alors égal à :

d'où :

$$
\begin{aligned}
s_{k} & =\frac{\mathrm{N}_{k}}{\mathrm{~N}^{\prime \prime}{ }_{k}} \\
\mathrm{P}_{k} & =\frac{s_{k}}{\mathrm{~N}} \times \frac{\mathrm{I}}{\mathrm{N}^{\prime}{ }_{k}}=\frac{\mathrm{I}}{s_{k} \times \mathrm{Q}^{\prime}{ }_{k}}
\end{aligned}
$$

A partir des différentes valeurs de $s_{k}$ (fixées à priori en fonction du cas) et de $\mathrm{Q}^{\prime}{ }_{k}$, nous avons pu calculer l'intensité de sélection $i$ grâce aux formules d'approximation données par Hastings (I955), plus précises que celle de Smith (I969) qui fait une approximation linéaire.

La valeur de $\mathrm{R}_{\mathrm{AA}}$ dépend du nombre de portées $k$ prises en compte dans l'index, du nombre de pleines sœurs $s_{k}$, et du nombre de portées $k^{\prime \prime}$ de la mère dont on connaît la moyenne. Le tableau 3 fournit les différentes valeurs adoptées pour chacune des situations mises en comparaison.

Dans cette analyse nous nous sommes intéressé à la taille de la portée à la naissance. Nous avons pour cela utilisé des estimées obtenues par analyse des performances enregistrées durant 1974, dans le troupeau de femelles A I077 lors de la première génération de sélection. Les valeurs de la variance phénotypique et de la répétabilité sont fixées à 6,54 et $0, \mathrm{r} 6$ respectivement. Nous avons considéré trois valeurs possibles de l'héritabilité du caractère : 0,05; 0,I0; 0,I5. Nous avons $\mathrm{pu}$ ainsi mettre en comparaison, vis-à-vis de l'espérance de progrès génétique annuel réalisable par la voie mère-fille, les ıo cas décrits au tableau 2. 


\section{2. - Résultats}

Les tableaux 3 et 4 et la figure I donnent pour Io cas les valeurs de l'espérance du progrès génétique annuel sur la taille de portée à la naissance, en fonction des 3 valeurs de l'héritabilité et des 2 possibilités sur le nombre de pleines sœurs utilisables par portée pour le renouvellement ( 2 ou 3 ). Les Io cas diffèrent par les nombres $k^{\prime}$ de portées de renouvellement, $k$ d'information, par le nombre $k^{\prime \prime}$ de mises bas de la mère et par l'intervalle de génération (tabl. 2 et 3 ). Le tableau 3 donne le gain annuel espéré sur le nombre de lapereaux nés par portée; le tableau 4 le donne en unité d'écart-type génétique.

TABLEAU 3

Valeurs des paramètres utilisés et espérance du progrès génétique annuel (lapereaux nés vivants par portée) selon les cas

Values of used parameters and expectation of annual genetic progress (rabbits born alive per litter) according the cases

\begin{tabular}{|c|c|c|c|c|c|c|c|c|c|c|c|}
\hline \multicolumn{2}{|r|}{ No du cas } & $\mathbf{I}$ & 2 & 3 & 4 & 5 & 6 & 7 & 8 & 9 & 10 \\
\hline \multicolumn{2}{|c|}{ 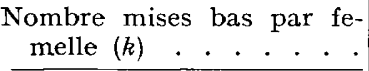 } & $\mathbf{I}$ & 2 & 2 & 3 & 3 & 3 & 4 & 4 & 4 & 4 \\
\hline \multicolumn{2}{|c|}{$\begin{array}{c}\text { Nombre mises bas de re- } \\
\text { nouv }^{\mathrm{t}}\left(k^{\prime}\right) \\
\end{array}$} & I & I & 2 & I & 2 & 3 & I & 2 & 3 & 4 \\
\hline \multicolumn{2}{|c|}{$\begin{array}{l}\text { Nombre mises bas de la } \\
\text { mère }(k) . \\
\end{array}$} & 3 & 4 & 4 & 5 & 5 & 5 & 6 & 6 & 6 & 6 \\
\hline \multirow{2}{*}{$h^{2}=0.15$} & $\begin{array}{l}R \text { max. } \\
3 \text { filles /M.B. }\end{array}$ & 1.00 & I. I I & I. 24 & I. I 3 & I. 30 & I. 29 & $0.8 \mathrm{I}$ & I.05 & I.09 & I.OI \\
\hline & $\begin{array}{l}\mathrm{R} \text { min. } \\
2 \text { filles /M.B. }\end{array}$ & 0.64 & 0.66 & I.06 & 0.60 & I. IO & I.I 7 & $0.3^{8}$ & 0.87 & 0.99 & 0.93 \\
\hline \multirow{2}{*}{$h^{2}=0.10$} & $\begin{array}{l}R \max . \\
3 \text { filles/M.B. }\end{array}$ & 0.67 & 0.76 & 0.84 & 0.77 & 0.88 & 0.87 & 0.55 & $0.7 \mathrm{I}$ & 0.73 & 0.68 \\
\hline & $\begin{array}{l}\mathrm{R} \text { min. } \\
2 \text { filles /M.B. }\end{array}$ & 0.43 & 0.45 & 0.72 & $0.4 \mathrm{I}$ & 0.75 & 0.79 & 0.34 & 0.59 & 0.67 & 0.63 \\
\hline \multirow{2}{*}{$h^{2}=0.05$} & $\begin{array}{l}R \max . \\
3 \text { filles /M.B. }\end{array}$ & 0.34 & 0.39 & 0.43 & $0.4^{\circ}$ & 0.46 & 0.45 & 0.29 & 0.37 & $0.3^{8}$ & 0.35 \\
\hline & $\begin{array}{l}\mathrm{R} \min . \\
2 \text { filles } / \mathrm{M} . \mathrm{B} .\end{array}$ & 0.22 & 0.23 & 0.37 & $0.2 \mathrm{I}$ & 0.39 & $0.4 \mathrm{I}$ & 0.13 & 0.31 & 0.34 & 032 \\
\hline
\end{tabular}

Ces tableaux font bien apparaître l'accroissement important de $\mathrm{R}$ progrès génétique attendu, lorsque l'héritabilité augmente (multiplication par presque 3 , lorsque $h^{2}$ passe de 0,05 à $0, I_{5}$ ); ou lorsque le nombre de filles de renouvellement passe de 2 à 3 par portée (l'accroissement relatif est surtout imfortant pour les faibles valeurs de $h^{2}$ ). Pour une valeur faible de $h^{2}$, égale à 0,05 , et pour 2 filles de renouvellement par portée seulement, on attend un progrès de 0,4 lapereaux par mise bas dans les situations $6,5,3$, on est à 0,3 pour les cas 9, Io, 8 , où 4 portées d'information sont attendues. Les progiès les plus faibles sont obtenus pour les situations où l'on renouvelle à l'aide d'une mise bas $(2, I, 4,7)$. Cette répar- 
tition se retrouve pour les valeurs $h^{2}=0$, Io et $h^{2}=0, I_{5}$, avec quelques petites modifications pour 3 filles dans chaque portée de renouvellement. Le cas 7 est toujours de loin le plus défavorable. Dans les cas 5 et 6 , l'on peut atteindre dans la meilleure des hypothèses un progrès de I,3 lapereau par portée.

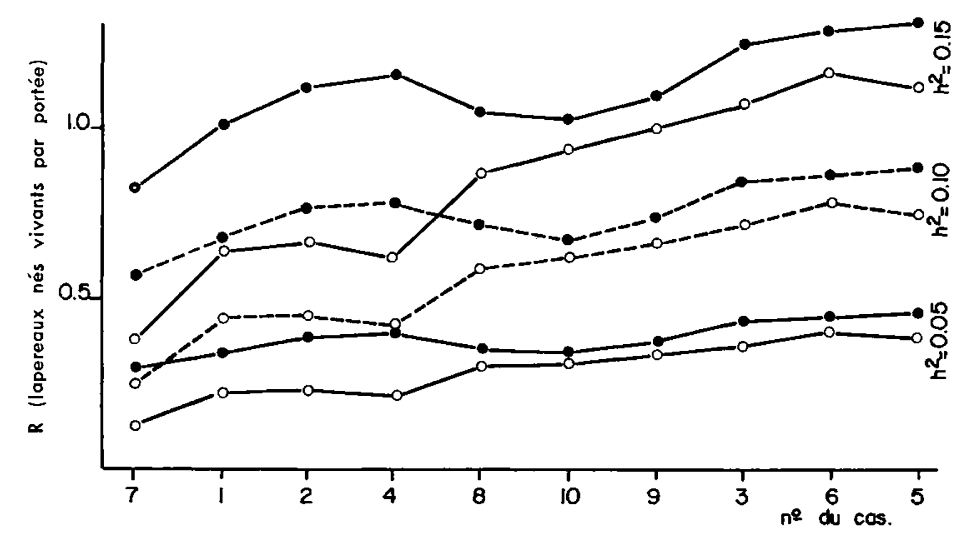

FIG. I. - Espérance de progrès génétique annuel selon les cas (*). Expectations of annual genetic progress according the cases

(*)

- 3 filles de renouvellement par portée.

$\bigcirc 2$ filles de renouvellement par portée.

L'espérance du progrès génétique peut être reliée aux intervalles de génération, indiqués au tableau 2 pour chacun des Io cas. Pour chacune des valeurs de $t$ $(6,8$, IO, I2 ou I 4 mois) nous avons porté sur la figure 2 les cas conduisant au progrès le plus grand, avec 2 ou 3 filles de renouvellement par portée de la mère. On voit qu'il y a un optimum de la valeur de $t$ pour un intervalle de génération entre 8 et Io mois. Il faut cependant noter que, pour ces mêmes intervalles de génération, certains cas sont très défavorables (cas $n^{0} 8$ et surtout 7 ).

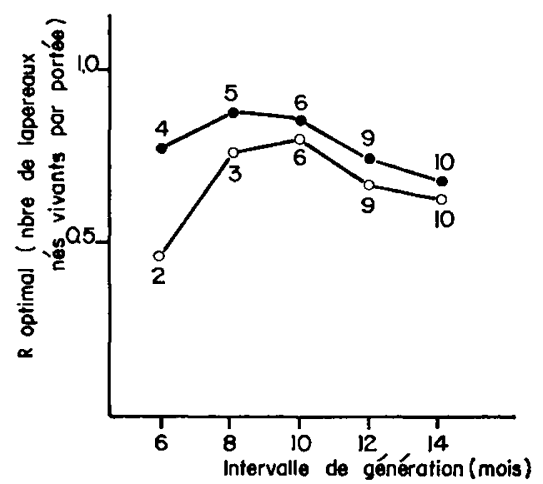

FIG. 2. - Évolution du progrès génétique annuel optimal en fonction de l'intervalle de génération pour une héritabilité de $h^{2}=$ o.1o $\left(^{*}\right)$

Evolution of annual optimal genetic progress in terms of generation interval for heritability $h^{2}=0.10$.

(*)

- 3 filles de renouvellement par portée.

2 filles de renouvellement par portée.

Les numéros représentent pour chaque point le cas donnant la plus grande espérance du progrès génétique (cf. tableau 2). 


\section{IV. - Discussion}

Celle-ci peut se situer à plusieurs points de vue puisqu'il s'agit d'une méthodologie expérimentale nouvelle proposée pour la sélection du lapin sur la taille de portée : sur le principe de la sélection en souche pure pour accroître les performances d'une lapine croisée; sur le plan d'accouplement et la méthode adoptés; sur les résultats attendus et les études ultérieures à réaliser.

Nous avons étudié ici la sélection de deux souches maternelles, les mâles de l'une (A I066) et les femelles de l'autre (A I077) devant donner en croisement une lapine à productivité numérique élevée, en optimisant le progrès génétique attendu en souche pure sur le seul caractère taille de portée à la naissance. Un calcul analogue conduit à des résultats comparables pour la taille de portée au sevrage. La connaissance précise des paramètres génétiques des effectifs par mise bas nés et sevrés dans ces populations, nous permettra de discuter la meilleure méthode de sélection pour augmenter la prolificité (directe, indirecte, sur les deux caractères à la fois). De même la pression de sélection à réaliser sur la vitesse de croissance dans ces souches sera à étudier en se plaçant dans un contexte plus global sur les possibilités de leur utilisation (souches paternelles et maternelles ou souches mixtes). On sait que les héritabilités en souche pure des caractères maternels sont faibles ce qui avait déjà été indiqué par RoLlins et CASADY (I964). Par contre ils bénéficient d'un effet d'hétérosis important de l'ordre de I5 p. Ioo et le sens du croisement ơ A I066 $\times$ \& A I077 semble actuellement être le meilleur, compte tenu de l'influence maternelle de la femelle A ro77 (Poujardieu et MatheRoN, I976). Enfin, les résultats préliminaires de F. Hulot et Matheron (non publiés) indiquent une complémentarité chez la lapine croisée entre le taux d'ovulation élevé de la souche A I 066 et le taux d'implantation et viabilité embryonnaire à I 6 jours élevés de la souche $\mathrm{A}$ I077. Il y aurait de plus hétérosis pour chacune de ces composantes de la taille de portée à la naissance. L'objectif, après avoir choisi ces deux souches d'après ces résultats expérimentaux est donc d'accrô̂tre les valeurs génétiques additives en souche pure, la mesure du progrès génétique réalisé se faisant par comparaison avec une population témoin (MatHeron et Chevalet, r977). Les résultats concernant les femelles issues du noyau de sélection et produisant dans des milieux différents (contrôle de performance en ferme) permettront d'optimiser à nouveau la sélection dans les souches de base. Ce n'est donc qu'à postériori que nous pourrons étudier l'efficacité d'une sélection récurrente qui pourrait présenter certains avantages (OROzCO, I973).

Le plan d'accouplement et la méthode sont analogues à ce que LERNER (I958) avait étudié pour la sélection des poules pondeuses et à la méthodologie de CocHEZ (Communication personnelle) également sur poule. L'expérimentation en sélection sur truies d'Ollivier (I972) se limitant à une sélection massale intra famille est plus comparable aux expériences de sélection sur animaux de laboratoire (souris). Dans le cas du lapin, contrairement à la poule, nous n'avons pas défini un plan d'accouplement différent du plan initial pour obtenir le cheptel de renouvellement (contraintes zootechniques). Les groupes de reproduction ne sont donc pas constitués par les reproducteurs sélectionnés une fois la sélection opérée. Ils sont constitués par les mâles et femelles de renouvellement, fils et filles de femelles sélectionnées d'après la valeur de leur indice. Le plan d'accouplement et de sélection retenu, en conservant toutes les origines paternelles, doit limiter la vitesse d'accroissement de la consanguinité et de réduction de la variabilité 
génétique dans la souche, bien que cela soit difficile à calculer du fait de la sélection. Une autre particularité de ce plan, est de croiser les origines paternelles des lapines mises en reproduction et les mâles (issus de ces mêmes origines) auxquels elles sont accouplées. Les croisements consanguins (frères $\times$ sœurs) sont ainsi en nombre limité et sont représentés dans la diagonale principale du tableau $\mathrm{I}$. Les effets des mâles sont distribués aléatoirement pour des femelles de toutes les familles. Cela offre l'intérêt supplémentaire de permettre l'estimation des paramètres génétiques de la population sélectionnée et d'estimer les progrès génétiques réalisés sans tenir compte des " effets paternels ". Le fait de procéder par générations séparées devrait conduire à une simplification sur les plans de l'interprétation génétique et de la sélection. Dans le cas du lapin, le taux de sélection dépend aussi de la fonte du cheptel de reproducteurs, ce que nous avons pris en compte dans l'expression de $\mathrm{P}_{k}$ en fonction de $\mathrm{Q}^{\prime}{ }_{k}$. Dans un système de reproduction continue, le cheptel serait constitué, au bout d'un certain temps, de lapines de différentes classes d'âge. La précision de l'estimation des valeurs génétiques additives serait plus faible, dans un troupeau de tel effectif limité, du fait du petit nombre de contemporaines. L'adoption de ce système de bandes séparées et la fonte du cheptel, permettent une utilisation génétique planifiée des troupeaux : les $k^{\prime}$ premières portées servent au renouvellement, les suivantes à produire les lapines (souche A I077) à utiliser en croisement pour l'obtention des lapines hybrides.

Sur le plan des résultats attendus en souche pure : les différents cas considérés (tabl. 2) tiennent compte des contraintes zootechniques. Le contrôle des tailles de portée à la naissance et au sevrage est routinier dans un élevage de lapins. Nous n'avons donc pas considéré de cas où le nombre de portées de renouvellement serait supérieur au nombre de portées d'information. Ollrvier (I974) dans le cas d'une sélection sur valeur phénotypique individuelle à partir de plusieurs enregistrements d'un caractère maternel d'héritabilité o.Io et de répétabilité o.I5 trouve la valeur optimale de progrès génétique annuel par la voie mère fille égale à 0.42 unités d'écart type génétique. Le tableau 4 montre qu'avec deux filles disponibles par portée pour effectuer le renouvellement, on obtient une valeur de $R$ variant de 0.92 à 0.30 unités d'écart type génétique. L'âge optimum d'une femelle le jour de son remplacement étant dans l'étude d'Ollivier de I7 mois (cela correspond à un intervalle de génération de $\mathrm{x} 2$ mois, cas $\mathrm{n}^{\circ}$ 9) on constate que l'on double la valeur de $R$ en utilisant un indice combiné $(R=0.82)$. Cette comparaison montre les possibilités de variations importantes de la réponse à la sélection selon le mode d'indexation (avec ou sans collatéraux) et en fonction de la démographie de la population (fonte de cheptel). Malgré les contraintes d'élevage introduites dans la présente étude, on peut aboutir à des résultats satisfaisants.

De façon générale, la méthode proposée qui consiste à indexer les lapines en tenant compte entre autre de la valeur phénotypique de sœurs contemporaines conduit à une amélioration notable. Les cas $1,2,4,7$, qui consistent à renouveler dans une seule portée donnent les résultats les plus défavorables, même si l'on tient compte des performances répétées (allongement, de plus, de l'intervalle de génération dans le cas $\mathrm{n}^{\circ} 7$ ), alors que l'intervalle de génération est minimum dans les cas I, 2, 4. Le cas Io est plus théorique que pratique (4 portées de renouvellement conduiraient à un décalage d'âges important entre les reproducteurs à la mise en place). Les cas 5 et 6 donnent, dans les conditions de l'étude, les progrès attendus les plus grands : 3 portées d'information, 2 ou 3 portées de renouvellement. Même pour une valeur de l'héritabilité aussi faible que 0.05 , on peut espérer un gain annuel de 0.4 lapereaux par portée. 
TABLEAU 4

Espérance de progrès génétique annuel en unité d'écart type génétique

Expectation of annual genetic progress (in genetic standard deviation unit)

\begin{tabular}{|c|c|c|c|c|c|c|c|c|c|c|c|}
\hline $\begin{array}{l}\text { Héri- } \\
\text { tabi- } \\
\text { lité }\end{array}$ & No du cas & I & 2 & 3 & 4 & 5 & 6 & 7 & 8 & 9 & Io \\
\hline \multirow{2}{*}{$\begin{array}{l}h^{2}= \\
\text { o.15 }\end{array}$} & R Max. & I. .00 & r.I I & I. 25 & I.I 4 & $\mathrm{I} \cdot 3 \mathrm{I}$ & I.30 & 0.82 & 1.06 & r.1o & 1.02 \\
\hline & R Min. & 0.64 & 0.67 & 1.07 & $0.6 \mathrm{r}$ & I.II & x.I 8 & $0.3^{8}$ & 0.88 & 1.00 & 0.94 \\
\hline \multirow{2}{*}{$\begin{array}{c}k^{2}= \\
0.10\end{array}$} & R Max. & 0.83 & 0.94 & I.O4 & 0.95 & 1.09 & I.07 & 0.68 & 0.87 & 0.90 & 0.84 \\
\hline & R Min. & 0.53 & 0.56 & 0.89 & $0.5 \mathrm{I}$ & 0.93 & 0.98 & $0.3^{\circ}$ & 0.73 & 0.82 & $0.7^{8}$ \\
\hline \multirow{2}{*}{$\begin{array}{c}h^{2}= \\
0.05\end{array}$} & R Max. & 0.60 & 0.68 & 0.76 & 0.69 & 0.80 & $0.7^{8}$ & $0.5^{\circ}$ & 0.64 & 0.66 & $0.6 \mathrm{I}$ \\
\hline & R Min. & 0.39 & $0.4 \mathrm{I}$ & 0.65 & 0.37 & 0.68 & $0.7^{2}$ & 0.22 & 0.54 & 0.60 & 0.57 \\
\hline
\end{tabular}

Enfin, les chiffres du tableau 3 et la figure I montrent bien l'intérêt de l'accroissement de la taille de portée pour permettre de mieux sélectionner sur celle-ci : le passage de 2 à 3 filles élevées par mise-bas laisse espérer un gain génétique nettement plus grand dans toutes les situations. On ne peut toutefois pas conclure à une plus grande efficacité de la sélection une fois celle-ci démarrée car elle devrait entre autre s'accompagner d'une modification de la variabilité génétique du caractère amélioré.

\section{V. - Conclusion}

La méthode de sélection que nous avons ici présentée et l'index employé sont classiques pour les caractères à faible héritabilité, il convenait cependant de discuter son application à la sélection du lapin, cela n'ayant jamais été fait auparavant. Un plan d'accouplement original permet de maîtriser l'augmentation du coefficient de consanguinité individuel (MATHERON et CHEvaleT, I977). Il conduit, en outre, à une randomisation de l'effet du mâle (effet paternel) sur la taille de portée de la femelle à laquelle il est accouplé, ce qui permet un classement des reproductrices indépendamment de cet effet.

La quantité optimale d'information qu'il faut attendre est, dans ce cas particulier de sélection de lapines, voisin de 3 portées par femelle et 4 à 6 pleines sœurs. Quelle que soit la quantité connue on peut toutefois noter (fig. I) que le renouvellement du troupeau doit se faire sur au moins 2 mises-bas. L'intervalle souhaitable 
entre 2 générations semble être compris entre 8 et ro mois. La méthode d'indexation basée sur la connaissance de 3 portées et sur le renouvellement du noyau de sélection avec les issues des 2 premières, conduit à la valeur de l'espérance du progrès génétique annuel par la voie mère-fille, la plus grande. Cette méthode peut, de plus, être mise en œuvre zootechniquement. S'il convient maintenant de mesurer le progrès génétique effectivement réalisé par comparaison avec la population témoin pendant quelques générations, dès que les paramètres génétiques de ces populations seront mieux connus des études plus globales de sélection pourront être réalisées : prise en compte des caractères de croissance, des résultats en croisement. Les résultats nécessaires devraient s'obtenir dans le cadre de la diffusion de ces reproducteurs auprès d'éleveurs du terrain et dans celui du contrôle de performances en ferme. A l'inverse, sur un plan biologique, il sera utile de préciser la variation génétique des composantes de la taille de portée (taux d'ovulation, taux d'implantation des blastocystes, viabilité embryonnaire) dans ces souches et d'en étudier les corrélations avec la taille de portée.

Rȩ̧u pour publication en juillet 1977.

\title{
Remerciements
}

Nous remercions C. Legault pour sa lecture critique et C. Chevalet pour sa contribution à l'amélioration de la rédaction du manuscrit.

\author{
Summary \\ Optimisation of genetic progress on the number of born \\ and weaned young rabbits
}

An original method of selection to increase litter size in the female rabbit is presented and discussed for a closed population with separated (non overlapping) generations. The method to index the females, based on the information of the female's first three litters and replacing from the animals produced on the first two litters, give the largest value of the annual genetic progress $(R)$ for litter size at birth (or at weaning). We find, in fact, $R=0.43$ for the genetic progress transmitted through the mother-daughter way, which is twice the value found in a population with overlapping generations, when the female's full-sib's and the mother's information is not taken under consideration. The tollowing aspects are discussed: pure line selection to improve, genetically, the hybrid female rabbit; determination of the lines used for selection; mating plans and selection method. All of them should allow the measurement of the genetic progress of the population, the estimation of genetic parameters and the study of the efficiency of a recurrent selection.

\section{Références bibliographiques}

FAlCoNer D. S., I960. Introduction to quantitative genetics. Oliver and Boyd London.

Hastings C. Jr., I955. Approximation for digital computers. Princeton University Press. 200 p. Lerner I. M., 1958. The genetic basis of selection. New York. John Wiley and sons Inc. 298 p. Lush J. L., I947. Family merit and individual merit as bases for selection. Amer. Nat., 81, 24I-26I, 362-379. 
Matheron G., Chevalet C., I977. Conduite d'une population témoin. Évolution à court terme du coefficient de consanguinité selon le schéma d'accouplement. Ann. Génét. Sél. Anim., I977, 9 (I), 3-I 5.

Moav R., I966. Specialised sire and dam lines. Anim. Prod., 8, 193-2 I I.

Ollivier L., I972. Note sur le taux optimum de renouvellement annuel des femelles dans la sélection du porc. Journées de la Recherche porcine en France, 13-67. Institut Technique du porc.

Ollivier L., I974. Optimum replacement rates in animal breeding. Anim. Prod., 19, $257-27$ I. Orozco F., I973. Additive gains in reciprocal recurrent selection. Genetics, 74, 204-205.

Osborne R., 1957. The use of sire and dam family averages in increasing the efficiency of selective breeding under a hierarchical mating system. Heredity, 11, 93-I 6.

Poujardieu B., Matheron G., r976. Hétérosis pour quelques caractères de reproduction chez le lapin. Analyse de plans de croisements. Bull. Techn. du Dép. Génét. Anim., 24, 69-77.

Poujardieu B., Rouvier R., I972. Optimisation du plan d'accouplement dans la sélection combinée. Ann. Génét. Sél. Anim., 3, 509-5r9.

Robertson A., I955. Prediction equations in quantitative genetics. Biometrics, 11, 95-98.

Rollins W. C., Casady R. B., I964. Heterosis in new zealand white $\times$ californian rabbit crosses.. J. Anim. Sci., 23, 853 .

Sмгтн C., 1964. The use of specialised sire and dam lines in selection for meat production Anim. Prod., 6, 337-344.

Sмгтн C., I969. Optimum selection procedures in animal breeding. Anim. Prod., 11, 433-442. 